\title{
Motional dispersions and ratchet effect in inertial systems
}

\author{
W. L. Reenbohn ${ }^{1}$, S. Saikia ${ }^{1,2}$, R. Roy ${ }^{3}$, Mangal C. Mahato ${ }^{1}$ \\ ${ }^{1}$ Department of Physics, North-Eastern Hill University, Shillong 793003, India \\ ${ }^{2}$ St.Anthony's College, Shillong-793003, India and \\ ${ }^{3}$ Women's College, Shillong-793003, India
}

\begin{abstract}
We obtain ratchet effect in inertial structureless systems in symmetric periodic potentials where the asymmetry comes from the nonuniform friction offered by the medium and driven by symmetric periodic forces. In the adiabatic limit the calculations are done by extending the matrix continued fraction method and also by numerically solving the appropriate Langevin equation. For finite frequency field drive the ratchet effect is obtained only numerically. In the transient time scales the system shows dispersionless behaviour as reported earlier when a constant force is applied. In the periodic drive case the dispersion behaviour is more complex. In this brief communication we report some of the results of our work.
\end{abstract}

Key Words: Ratchet current,inhomogeneous systems, underdamped Langevin equation, coherent motion

\section{INTRODUCTION}

The phenomenon of obtaining net unidirectional current in a periodic potential without the application of any time averaged external field is termed as ratchet effect[1]. This is necessarily a non-equilibrium phenomenon and has been investigated extensively in systems where damping to periodic motion is large. Also, in majority of the investigations the potential is considered asymmetric (and hence the name ratchet). In order to obtain ratchet effect in asymmteric potentials the system is rocked[2] periodically 
(rocked ratchet) or the potential amplitude is changed[3] dichotomously, periodically or randomly, (flashing or fluctuating-potential ratchets). The effect can also be obtained if the system is driven periodically but time asymmetrically in such a way that the total applied force per period is zero[4]. In such systems the condition for asymmetric periodic potential can be waived. All these popular models consider the systems to be homogeneous where the friction coefficient is taken to be constant in space and the temperature is maintained uniform. In the present work we consider an underdamped system and the periodic potential is taken to be symmetric. However, the friction coefficient is considered to vary periodically in space similar to the potential but with a phase difference. Also, the system is driven periodically symmetrically about zero. The overdamped case of the problem has been studied earlier [5].

Since the system asymmetry is solely due to non-uniformity of friction coefficient the expected net particle current in such a system may be weak [6]. Nevertheless, the physical explanation for asymmetric particle current can be easily understood. In fig. 1 the periodic potential $V(x)=-\sin (x)$ and the friction coefficient $\gamma(x)=$ $\gamma_{0}[1-\lambda \sin (x+\phi]$ are plotted together with $\lambda=0.9$, phase difference $\phi=0.35$ and $\gamma_{0}=1$. In the figure a period of the potential is shown divided into two halves one with friction coefficient small $(<1.0)$ and the other with large $(>1.0)$ friction coefficient. The effect of $\phi$ on this division is clearly visible: The friction is more on the left of the potential peak position than on the right.

The physical explanation for the possibility of obtaining ratchet effect in this inhomogeneous system can be given in two ways. Firstly, since in the static situation the position probability distribution is independent of the profile of the friction coefficient, the distribution will be Gaussian-like and symmetric about the minima of the potential. However, the situation changes in the dynamic case. Because of the higher friction on the left of the potential peak position the particle spends more time there during its motion. And hence the effective position probability distribution is skewed with higher probability on the left than on the right of the potential peaks as though 


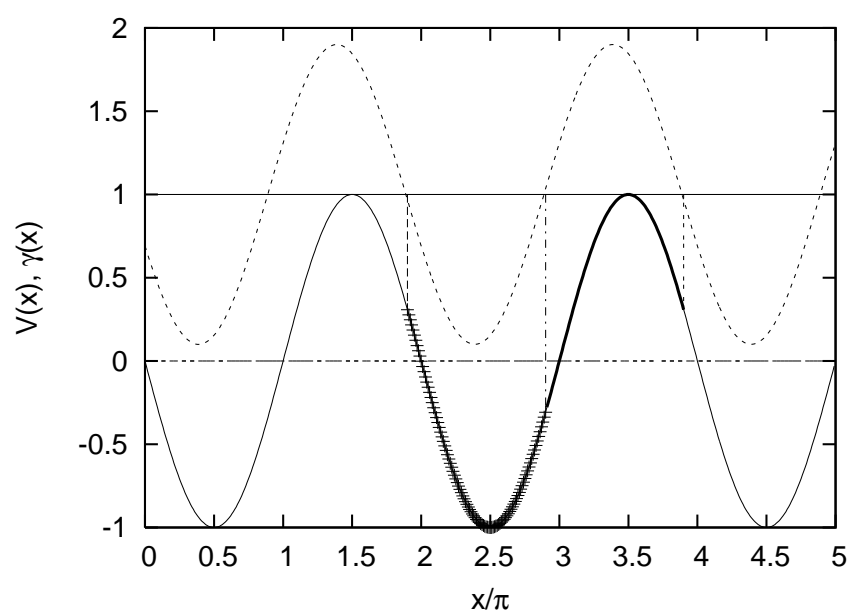

FIG. 1: Plot of potential $V(x)$ (solid line) and the friction coefficient $\gamma(x)$ (dashed line). The portion of a period of $V(x)$ where $\gamma(x)<1$ is shown hatched and the portion where $\gamma(x)>1$ is shown by a thick line.

a static constant force has been applied[5] in the left (negative) direction. Thus, one would expect a negative average particle current even without the application of net external field. Secondly, since the particles tend to spend more time on the left of the peak it will absorb more thermal energy from the bath and hence effectively it will be at a higher temperature on the left side of the potential peaks. Higher effective temperature on the left leads to higher probability of thermally assisted passage from the left of the potential peak to the right giving rise to a net current in the right (or positive) direction. These two opposing effects in the nonequilibrium situation may lead to competition in deciding the direction of the ratchet current depending on the temperature. These two competing effects could even lead to ratchet current direction reversals as will be revealed by our numerical results.

The underdamped systems are difficult to study (analytically as well as numerically) but provide interesting results. If the particle is initially put at the bottom of one of the wells of the potential (not with great initial velocity) it will get out of the well due to thermal effect (Kramers-like behaviour). However, once out of the well it will 
roll down from the top to the bottom of the next well of the tilted periodic potential without much frictional loss of energy and hence may continue to move along for a long distance (more than one period) before it again gets trapped in a distant well. From there it will again get out due to thermal effects and the process will keep repeating. The distribution of travel distances between two consecutive haltings is itself quite interesting to study. When taken an ensemble of such particles they may collectively show coherence properties in the intermediate time scales, where position dispersions $<(\Delta x(t))^{2}>=<x(t)^{2}>-<x(t)>^{2}$ become constant in time for a brief period. This is how the long time steady state behaviour $\left\langle(\Delta x(t))^{2}>\sim t\right.$ is bridged with the very short time behaviour $<(\Delta x(t))^{2}>\sim t^{2}$. This result has been reported recently for a constant applied force[7]. In the periodically driven case, however, the system shows more complex behaviour to be described as we proceed.

Obtaining analytical solutions of the Langevin equation for underdamped inhomogeneous (space-dependent friction) systems subjected to constant uniform force field in the steady state is in principle a straightforward extension of the matrix continued fraction method (MCFM) initially developed by Risken[8]. Our extended formalism, however, yields stable solutions only in a limited range of the applied force, especially for small friction coefficients. It turns out that the range of the applied force where MCFM yields sensible results is the one relevant to our discussion. In section II, we simply describe how to calculate the mean particle velocity in the steady state. However, it has not yet been possible to write down a similar expression for the solution of the Langevin equation when the system is subjected to a time varying external field. We, therefore, resort to numerical methods. The efficacy of the numerical method is established by comparing the results so obtained with the MCFM result in the adiabatic case (Fig.2). The numerical results along with the MCFM (adiabatic case) results are presented in section III. In section IV we discuss the salient features of our work to conclude. 


\section{THE METHOD}

Consider a particle of mass $m$ moving in a periodic potential $V(x)=-V_{0} \sin (k x)$ in a medium where the particle experiences friction with coefficient $\gamma(x)=\gamma_{0}(1-$ $\lambda \sin (k x+\phi))$ with $\lambda<1$. The motion can be described by the following Langevin equation:

$$
m \frac{d^{2} x}{d t^{2}}=-\gamma(x) \frac{d x}{d t}-\frac{\partial V(x)}{\partial x}+F(t)+\sqrt{\gamma(x) T} \xi(t),
$$

where $T$ is the temperature in energy units in terms of $k_{B}$, the Boltzmann constant and $F(t)$ is the externally applied field. $\xi(t)$ is the fluctuating force term with $<\xi(t)\rangle=0$, and $<\xi(t) \xi\left(t^{\prime}\right)>=2 \delta\left(t-t^{\prime}\right)$. In dimensionless units with $m=1, V_{0}=1, k=1$, the Langevin equation reduces (reduced variables denoted by the same symbols) to

$$
\frac{d^{2} x}{d t^{2}}=-\gamma(x) \frac{d x}{d t}+\cos x+F(t)+\sqrt{\gamma(x) T} \xi(t),
$$

where $\gamma(x)=\gamma_{0}(1-\lambda \sin (x+\phi))$ and with similar noise statistics for the reduced $\xi(t)$ as earlier. Our purpose is to calculate $x(t)$ and $v(t)$ by solving the Langevin equation. As mentioned earlier, so far no analytical solution could be found even for the average velocity of the particle when the external force $F(t)$ is time varying. However, when $F(t)=F_{0}$, a constant, it is straightforward to extend the MCFM to obtain the drift velocity in the steady state situation. The method involves solving the Fokker-Planck equation

$$
\frac{\partial W(x, v, t)}{\partial t}=\mathcal{L}_{F P} W(x, v, t)
$$

corresponding to the Langevin equation (2), where the FP operator

$$
\begin{aligned}
\mathcal{L}_{\mathcal{F P}}= & -v \frac{\partial}{\partial x}+\gamma_{0}(1-\sin (x+\phi)) \frac{\partial}{\partial v} v-\left(\cos x+F_{0}\right) \frac{\partial}{\partial v}+ \\
& \gamma_{0} T(1-\lambda \sin (x+\phi)) \frac{\partial^{2}}{\partial v^{2}} .
\end{aligned}
$$

In the MCFM, the distribution $W(x, v, t)$ is expanded in terms of the function $\psi_{n}$,

$$
W(x, v, t)=(2 \pi T)^{\frac{-1}{4}} e^{\frac{-v^{2}}{4 T}} \sum_{n=0}^{\infty} C_{n}(x, t) \psi_{n},
$$


where $\psi_{n}=\frac{\left(b^{\dagger}\right)^{n} \psi_{0}}{\sqrt{n !}}$ are the eigen functions of the operator $\gamma(x) b^{\dagger} b$ and

$$
\psi_{0}=(2 \pi)^{\frac{-1}{4}} T^{\frac{-1}{4}} e^{\frac{-v^{2}}{4 T}} .
$$

$b=\sqrt{T} \frac{\partial}{\partial v}+\frac{1}{2} \frac{v}{\sqrt{T}}, b^{\dagger}=-\sqrt{T} \frac{\partial}{\partial v}+\frac{1}{2} \frac{v}{\sqrt{T}}$. In the steady state, the Fokker-Planck equation reduces to

$$
n \gamma(x) C_{n}(x)+\sqrt{n+1} D C_{n+1}(x)+\sqrt{n} \hat{D} C_{n-1}(x)=0,
$$

where $D=\sqrt{T} \frac{\partial}{\partial x}$ and $\hat{D}=\sqrt{T} \frac{\partial}{\partial x}+\frac{-\cos x-F}{\sqrt{T}}$. This is a series of recurring equations for $n=0,1,2, \ldots, N$. This series is truncated at $n=N$ which depends on the value of $\gamma, F$, etc and can be quite large. Since the potential as well as the friction coefficient are periodic, the mean particle velocity $\langle v\rangle$ is given by $\int_{-\infty}^{\infty} d v \int_{0}^{2 \pi} d x W(x, v) v$ which turns out to be a constant from the first, $n=0$, of the series of the equations (7). Solving these simultaneous equations to obtain $C_{n}(x)$ is not straightforward, however, and one may not obtain any stable solution for all values of $\gamma_{0}$, and $F$.

For the time dependent external force fields, one necessarily have to use numerical methods to solve the Langevin equation. For homogeneous inertial systems but with asymmetric periodic potentials, the ratchet effect has been investigated recently by solving the Langevin equation numerically[9]. In the present work we have used stochastic form of the $4^{\text {th }}$ order Runge-Kutta method. Numerically, one can calculate position dispersion at various times and also the distribution of instantaneous velocities for the entire period of motion. The drift velocity can be obtained either by taking the mean position at a large time and dividing by the time: $\langle v\rangle=\lim _{t \rightarrow \infty} \frac{\langle x(t)\rangle}{t}$ or $\langle v\rangle=\int_{-\infty}^{\infty} v \rho(v) d v$, where $\rho(v)$ is the velocity distribution. The $\langle\ldots\rangle$ in $<v>=\lim _{t \rightarrow \infty} \frac{\langle x(t)>}{t}$ is the average evaluated over several realizations of the stochastic run whereas in the later case the (instaneous) velocity distribution is obtained for several realizations of the entire stochastic run. In the following we present results of our calculation first for the adiabatic case and then for the time-varying (finite frequency) field case. 


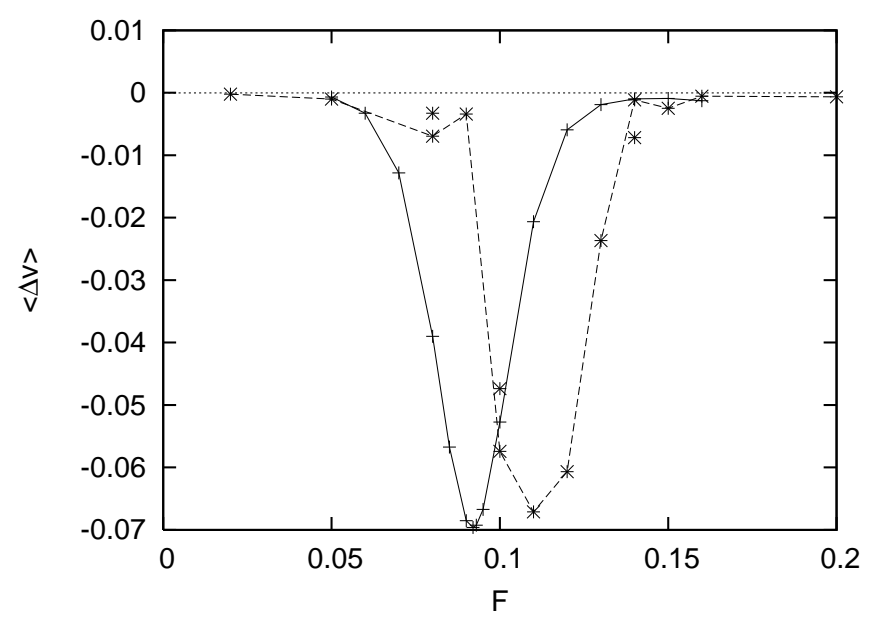

FIG. 2: Shows the ratchet current $\langle\Delta v\rangle$ as a function of $F$ using MCFM ( $\dagger$ joined by dashed lines) and the Langevin simulation method ( $*$ joined by dashed lines) for $\gamma=0.035$, $T=0.4, \phi=0.35$. The dotted line for zero current is shown just to guide the eye.

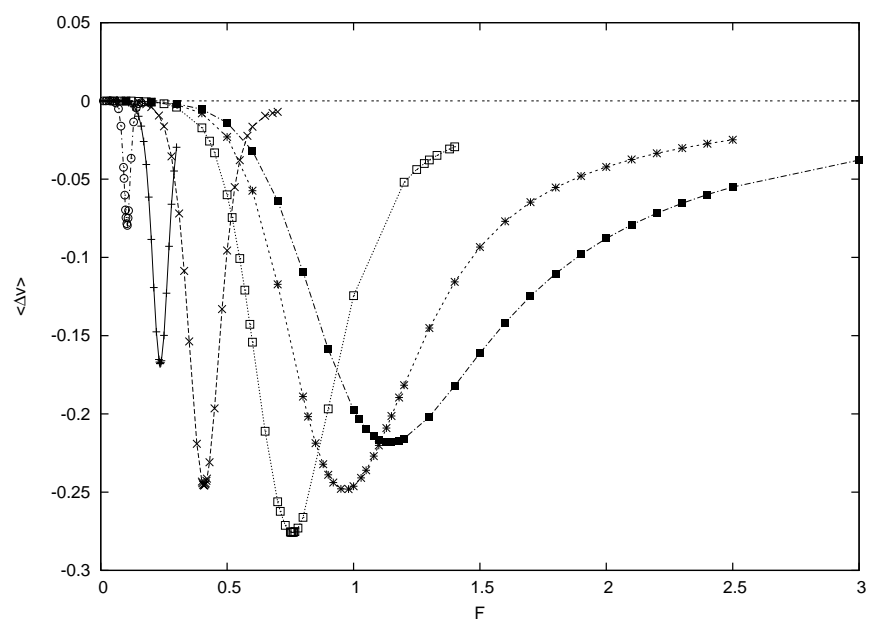

FIG. 3: The variation of $\langle\Delta v\rangle$ as a function of $F$ is shown for $\gamma=0.04(\circ), 0.1(+), 0.2$ (x), 0.5 (open square), $0.75(*)$ and 1.0 (solid squares) 


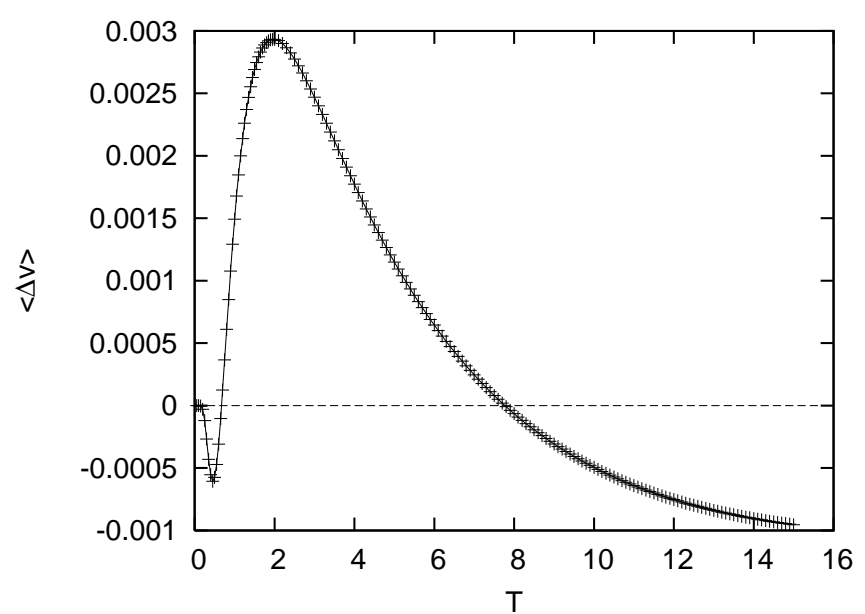

FIG. 4: Shows variation of $\langle\Delta v\rangle$ as a function of temperature $T$ for $\gamma_{0}=0.1$ and $F_{0}=0.1$.

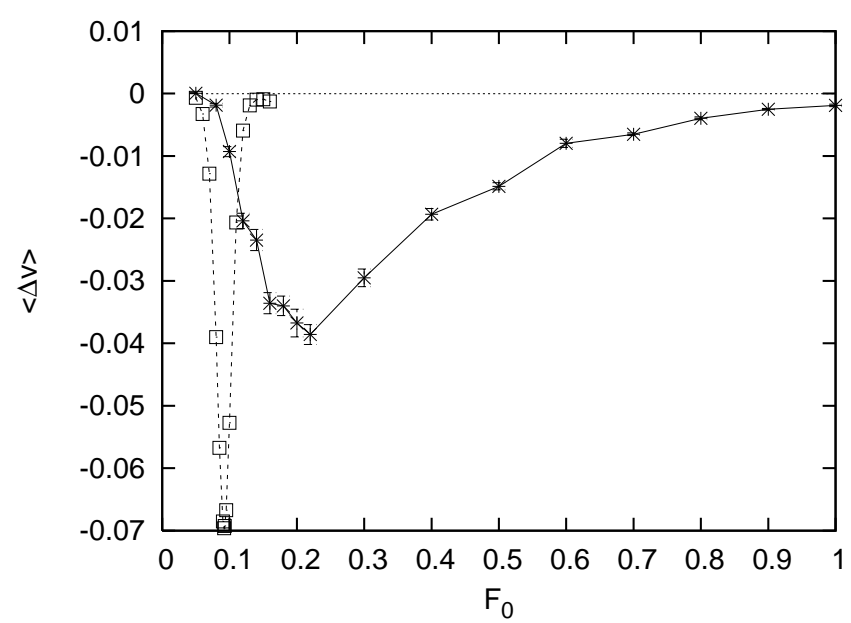

FIG. 5: The ratchet current $\langle\Delta v\rangle$ when the system was driven by a square-wave field of amplitude $F_{0}$ are shown $(+)$ with errorbars for $\gamma_{0}=0.035$ and $T=0.4$. The curve (with open box) is for the adiabatic case for the same $\gamma_{0}$ and $T$ 


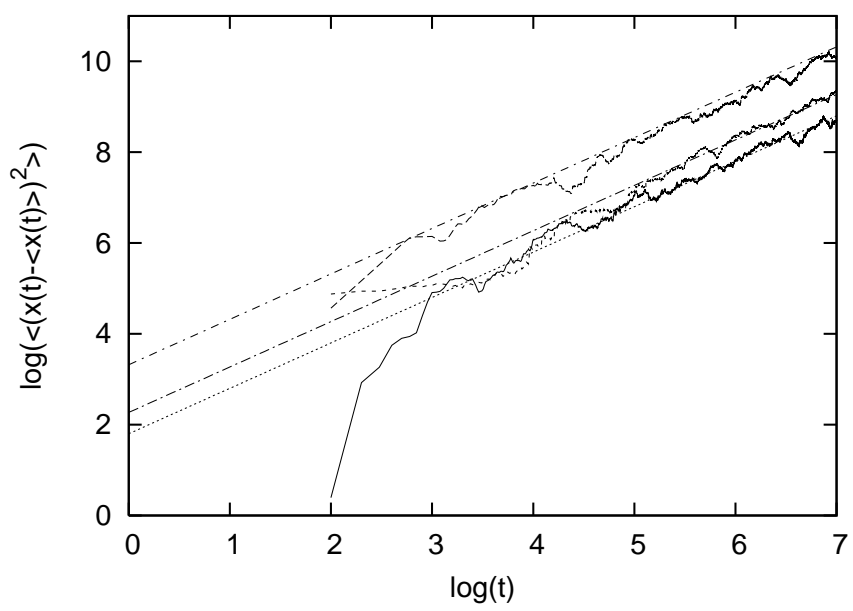

FIG. 6: The position dispersions $\left\langle(\Delta x)^{2}>\right.$ are plotted as a function of time $t$ for $F_{0}=0.08$ (solid line), 0.3 (dashed line) and 0.6 (small dashed line) for $\gamma=0.035$ and $T=0.4$. The straight lines are drawn to indicate the average slope equal to 1 and intercepts 1.8, 3.2 and 2.27 respectively for the above $F_{0}$ values of the curves at large $t$ limit.

\section{NUMERICAL RESULTS}

\section{A. Adiabatic Case}

The matrix continued fraction method is used to calculate the drift velocity, (and hence mobilities), the average potential and kinetic energies, etc, as a function of $\gamma_{0}, F_{0}$ and T. However, in this paper we present only the drift velocities to illustrate the ratchet effect in inhomogeneous (non-uniform friction) inertial systems. As will be detailed in Ref [10] the expansion coefficients $C_{n}(x)$ are expressed in terms of their Fourier components $C_{n}^{q}$ which form column matrices $\mathbf{C}_{\mathbf{n}}$ for each n. By a clever transformation $\mathbf{C}_{\mathbf{n}}$ and $\mathbf{C}_{\mathbf{n}-\mathbf{1}}$ are related. $\mathbf{C}_{\mathbf{1}}$ being constant it can be found through the normalization condition, $1=\int_{-\pi}^{\pi} C_{0}(x) d x$ and by evaluating the relational matrix (between $\mathbf{C}_{\mathbf{0}}$ and $\mathbf{C}_{\mathbf{1}}$ ) which depends on the Fourier componets of $V(x)$ and $\gamma(x)$ and $F$. A typical mean velocity evaluation may require $N$ as large as 1000 and the number of Fourier components typically as large as 30 , if at all a stable solution is to be found. The drift 


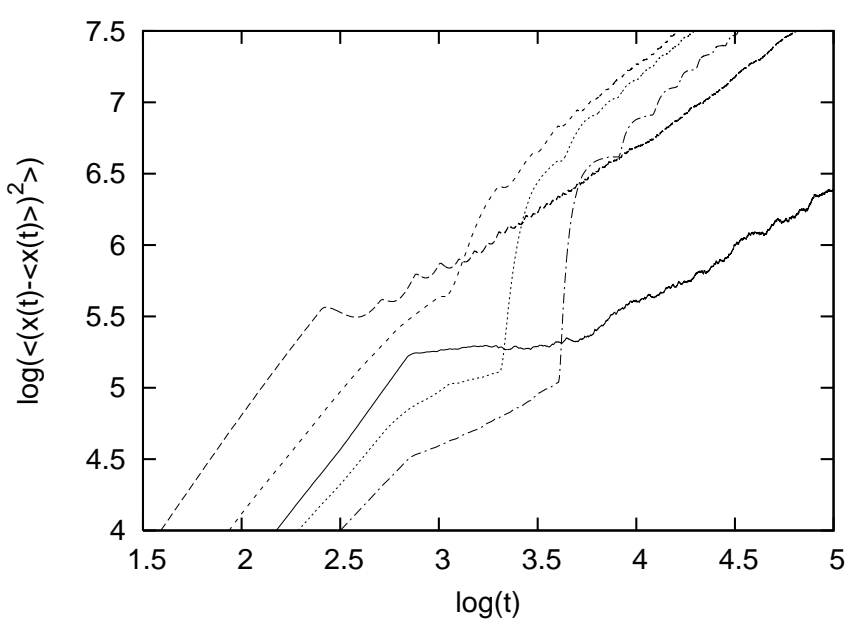

FIG. 7: The position dispersions $<(\Delta x)^{2}>$ as a function of time $t$ are shown for the square wave drive amplitude $F_{0}=0.2$ but with periods 500 (long dashed line), 2000 (small dashed line), 4000 (dotted line), and 8000 (dashed-dotted line) for $\gamma=0.035, T=0.4$, and $\phi=0.35$. The dispersions when a constant force $F_{0}=0.2$ is applied is shown by the solid line. This curve is analogous to the homogeneous case of [7].

velocity, obtained by taking the sum of mean velocities corresponding to the applied force $F=\left|F_{0}\right|$ and $F=-\left|F_{0}\right|$, as a function of $F$ is shown in Fig.2 for $\gamma_{0}=0.035$. This case of calculating the drift velocities or the ratchet currents, corresponding to the effect of application of a zero averaged drive field of frequency $\omega \rightarrow 0$, is referred to as the adiabatic case. In the same figure we have also plotted drift velocities obtained by numerically solving the Langevin equation. Here we have presented the sum $\Delta \bar{v}$ of $\bar{v}$ for $F= \pm\left|F_{0}\right|$, where $\bar{v}=\lim _{t \rightarrow \infty} \frac{x(t)}{t}$ without the ensemble averaging. The ensemble averaging, however, does not qualitatively change the nature of the graph. The comparison of the two graphs shows that the Langevin simulation yields qualitatively similar result to what is obtained using MCFM. However, the peak ratchet currents occur at slightly different values of $F$, both being at $F \ll 1$. Importantly, the ratchet current, in this adiabatic limit, results only in a very small range of $F$. In the following, for the adiabatic case, we present results obtained from MCFM calculations only. 
From these calculations we find that the ratchet current do not change its qualitative behaviour for different $\gamma_{0}$. However, the narrow peaks occur at values of $F$ that depend on the values of $\gamma_{0}$ and $T$. As $\gamma_{0}$ is increased the peak position gradually shifts towards larger $F$ and also the peak width increases. The largest peak for $T=0.4$, occurs for $\gamma_{0}=0.4$. Also, at $T=0.4$ the ratchet current is in the negative direction (Fig.3). However, there is a distinct possibility of current reversals as a function of temperature $T$ as is seen in Fig.4. The figure also shows clearly that the net current could also peak as a function of the temperature $T$. It is worth reminding that the current $\langle\Delta v\rangle$ here (in the adiabatic case) is just the sum of the average currents corresponding to $F= \pm|F|$. The average currents corresponding to $\pm|F|$ need not, however, be nonmonotonous individually. From Fig.3, there is a very clear indication that $\langle\Delta v(\gamma)>$ will also be non-monotonous.

Since there is no way we can calculate analytically the average currents for time varying fields we present (numerical) Langevin simulation results for the non-adiabatic case.

\section{B. Non-adiabatic case}

In the finite (albeit small) frequency drive case, we consider a square wave force field where $F(t)=F_{0}$ for time interval $T_{\Omega}=1000$ and in the next $\left(T_{\Omega}=1000\right)$ interval[11] the force is taken as $-F_{0}$ and repeated the procedure for a large number of periods (typically equal to 5,000 complete cycles) and the mean velocity is calculated. An average of this is taken over several realizations (typically 20), thus evaluating the ensemble averaged mean velocity or the net (ratchet) current. The obtained steady state current as a function of $F_{0}$ is plotted in Fig. 5 for $\gamma_{0}=0.035$. For comparison the current obtained in the adiabatic case is plotted together. The range of $F$ for which ratchet current can be obtained has broadened considerably in comparison to the adiabatic case. The value of $F$ at which the peak current is obtained has also 
shifted to a higher value. In Fig.6, one can see that around $t \sim 10^{7}$, the position dispersion $<(\Delta x)^{2}>=<x^{2}(t)>-<x(t)>^{2} \sim t$ hinting at the approach to the diffusive steady state situation.

Fig.6 with $<(\Delta x(t))^{2}>\sim t$ provides a method to calculate the diffusion constant $D$ in the large time limit. It is interesting to observe that in this limit $<(\Delta x)^{2}>$ shows a peaking behaviour as a function of F. For $\gamma=0.035$, the peak ratchet current occurs close to where $<(\Delta x)^{2}>$ is maximum. It would, therefore, appear that ratchet current in this case is dispersive and not coherent. However, when the relative dispersion is plotted as a function of $F, \frac{\left\langle(\Delta x)^{2}\right\rangle}{\langle x\rangle^{2}}$ shows small values $<1$ in the range of $F$ where ratchet current is obtained. Thus, the obtained ratchet current is, indeed, a result of coherent motion of particles. Equivalently, the related Peclet number $P e$ which is a measure of coherence of motion, turns out to be much larger than 2 indicating the particle motion giving rise to steady state ratchet current to be coherent. The motion at the intermediate (transient) time scales, however, shows a very different nature.

As mentioned earlier, Lindenberg, et. al.|7] have shown that the motion of an ensemble of non-interacting particles in a periodic potential together with a uniformly applied constant force showed dispersionless behaviour at intermediate time scales. They have set the time interval $\left[t_{\text {min }}<t<t_{\max }\right]$ for a given applied force $F_{0}$ during which the system shows such an interesting behaviour. This behaviour is seen only for a certain range of $F_{0}$ values. A typical graph of $\left\langle(\Delta x(t))^{2}>\right.$ on log-log scale is presented numerically in Fig.7. In this figure are also shown the effect of frequency of the dichotomously time varying (square wave) applied field of same amplitude $F_{0}=0.2$. If the half period of the applied field over which the field remains constant before its sign is changed, $T_{\Omega}<t_{\min }$, then after $T_{\Omega}$ the dispersion shows a dip before again picking up. This is repeated in every $T_{\Omega}$. If, however, $T_{\Omega}>t_{\min }$ at $t=t_{\min }$ the slope of $\left\langle\Delta x^{2}\right\rangle$ changes abruptly. At $t=T_{\Omega},<(\Delta x)^{2}>$ begins to increase sharply before it appears to level itself up till it reaches $t=2 T_{\Omega}$. The behaviour shown between $T_{\Omega}$ and $2 T_{\Omega}$ is seen to repeat every $T_{\Omega}$ for a long time. The frequency dependence of applied force on 
the dispersive behaviour of particle motion shows interesting possibilities of dispersion reduction as well as brief dispersionless motion.

\section{DISCUSSION AND CONCLUSION}

The present work gives numerical evidence of the possibility [4] of obtaining ratchet current in an inertial inhomogeneous symmetric periodic potential system subjected to a symmetrically driven periodic external field. The nonuniform temperature case has been investigated earlier by Blanter, et al.[12]. We have calculated the net current from the average distance travelled after a long time. However, almost the same current is obtained from the (instantaneous) particle-velocity distribution over the entire sojourn time of the particles. But we find that the efficiency with which useful work can be derived from such a system is quite low and falls in the subpercentage range. This could be because the system inhomogeneity considered here is a feeble cause to generate net current without the asymmetric support of external forcings. Preliminary work, however, shows that the magnitude of ratchet current can be increased dramatically if, instead of time symmetric drive, time asymmetric drive with zero time averaged force per period is used. Similar effect for overdamped case has been reported earlier [13].

The velocity distribution in this case shows an overwhelming asymmetry in favour of particle motion in the direction of current in contrast to the symmetric drive case where it shows almost symmetric velocity distribution. The width of the velocity distribution [9] increases with the amplitude of the periodic driving. In conclusion, even though system inhomogeneity is a weak ingredient to obtain ratchet current it is a distinctive way of obtaining such a current.

\section{ACKNOWLEDGEMENT}

MCM thanks A. M. Jayannavar for discussions. MCM and WLR acknowledge partial financial support from BRNS, DAE, Govt. of India. RR wishes to thank DST, 
Govt. of India for financial assistance (SR/FTP/PS-33/2004).

[1] P. Reimann, Phys. Rep. $\underline{361}$, 57(2002). Apart from the extensive references cited here, we give below only few representative references.

[2] M. O. Magnasco, Phys. Rev. Lett.1, 1477(1993).

[3] J. Prost, J. F. Chauwin, L. Peliti, and A. Ajdari, Phys. Rev. Lett.72, 2652(1994).

[4] M. C. Mahato and A. .M. Jayannavar, Phys. Lett. A $\underline{209}$, 21(1995); D. R. Chialvo and M. M. Millonas, Phys. Lett. A $\underline{209}, 26(1995)$.

[5] M. Büttiker, Z. Phys. B $\underline{68}$, 161(1987); M. C. Mahato, T. P. Pareek and A. .M. Jayannavar, Int. J. Mod. Phys. B $\underline{10}, 3857(1996)$.

[6] M. C. Mahato, Indian J. Phys $\underline{78}, 693(2004)$.

[7] K. Lindenberg, J. M. Sancho, A. .M. Lacasta, and I. M. Sokolov, Phys. Rev. Lett. $\underline{98}$, 020602(2007).

[8] H. Risken, The Fokker-Planck Equation, Springer Verlag, Berlin, 1989; H. Risken and H. D. Vollmer, Z. Phys. B $\underline{35}$, 177(1979).

[9] L. Machura, M. Kostur, P. Talkner, J. Luczka, F. Marchesoni, and P. Hänggi, Phys.

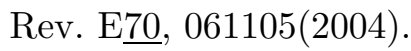

[10] W. L. Reenbohn, S. Saikia, and M. C. Mahato, unpublished.

[11] For an equivalent RCSJ model of Josephson junctions the characteristic plasma frequency $\omega_{J}$ will be about $10^{3}$ times larger than the drive field frequency corresponding to $T_{\Omega}=1000$.

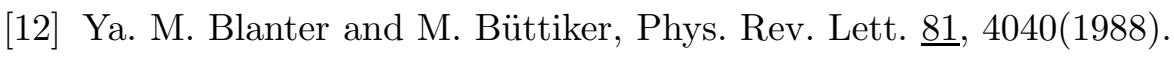

[13] D. Dan, M. C. Mahato, and A. M. Jayannavar, Physica A296, 375(2001); Phys. Rev. $\mathrm{E} \underline{63}, 056307(2001)$. 